\title{
Approximate identities on some homogeneous Banach spaces
}

\author{
F. Andreano - M. A. Picardello
}

Received: 11 March 2008 / Accepted: 3 March 2009 / Published online: 25 March 2009

(C) Springer-Verlag 2009

\begin{abstract}
We study convergence of approximate identities on some complete seminormed or normed spaces of locally $L^{p}$ functions where translations are isometries, namely Marcinkiewicz spaces $\mathcal{M}^{p}$ and Stepanoff spaces $\mathcal{S}^{p}, 1 \leqslant p<\infty$, as well as others where translations are not isometric but bounded (the bounded $p$-mean spaces $M^{p}$ ) or even unbounded $\left(M_{0}^{p}\right)$. We construct a function $f$ that belongs to these spaces and has the property that all approximate identities $\phi_{\varepsilon} * f$ converge to $f$ pointwise but they never converge in norm.
\end{abstract}

Keywords Approximate identities - Locally $L^{p}$ functions - Bounded $p$-means . Marcinkiewicz spaces - Stepanoff spaces - Wiener amalgams · Herz spaces . Continuity of translation $\cdot$ Semi-normed function spaces $\cdot$ Convolution operators

Mathematics Subject Classification (2000) Primary 41A65;

Secondary $42 \mathrm{~A} 85 \cdot 46 \mathrm{E} 30$

Communicated by K. H. Gröchenig.

F. Andreano $(\varangle)$

Department of Computer Sciences, University of Insubria,

Via Mazzini 5, 21100 Varese, Italy

e-mail: federica.andreano@uninsubria.it

M. A. Picardello

Department of Mathematics, University of Rome "Tor Vergata",

Via Ricerca Scientifica, 00133 Rome, Italy

e-mail: picard@mat.uniroma2.it 


\section{Introduction: some classical spaces of bounded $L^{p}$-means}

Convolution operators are usually considered on subspaces of $L^{p}$ spaces, but they have also been studied on spaces of locally summable functions, or more generally functions that belong to $L^{p}$ on all compact subsets of $\mathbb{R}$. Of course, the lack of summability on the whole of $\mathbb{R}$ makes this task considerably more delicate. Here we consider several locally $L^{p}$ normed (or semi-normed) spaces on $\mathbb{R}$, each defined by the condition that the $L^{p}$-averages on compact sets be bounded, in an appropriate sense.

An approximate identity is a one-parameter family of convolution operators with kernel $\phi_{\varepsilon}(x)=\frac{1}{\varepsilon} \phi\left(\frac{x}{\varepsilon}\right)$, where $\varepsilon>0, \phi \geqslant 0$ and $\phi \in L^{1}(\mathbb{R})$ or $L_{w}^{1}(\mathbb{R})$ (respectively) with norm 1.

On every $L^{p}$ space, approximate identities are well known to converge in norm and pointwise almost everywhere. The aim of this paper is to show that this behaviour of approximate identities depends not only on $L^{p}$ finiteness on compact sets, but also on the decay at infinity, even when the approximate identities are compactly supported.

In this section, we introduce several well known spaces of functions with bounded $L^{p}$ averages but only locally $L^{p}$ : the (semi-normed ) Marcinkiewicz spaces $\mathcal{M}^{p}$, its Banach quotients $\widetilde{\mathcal{M}^{p}}$, the Stepanoff spaces $\mathcal{S}^{p}$, the bounded $p$-mean spaces $M^{p}$ with positive lower bound on the mean width, and the corresponding spaces $M_{0}^{p}$ defined by means over intervals whose width may tend to zero.

In the next section we collect various results on (lack of) continuity of translations and norm convergence of approximate identities in these spaces. It then follows from Propositions 2.5 and 2.6 below that approximate identities do not converge in the norm of $\mathcal{M}^{p}, \widetilde{\mathcal{M}}^{p}, \mathcal{S}^{p}$ and $M^{p}$. Remark 2.4 suggests that the same should be true for $M_{0}^{p}$. General sufficient conditions for pointwise convergence of approximate identities have been obtained in [9] for a class of spaces equivalent to Stepanoff spaces (namely, Wiener amalgams); moreover, the spaces $M^{p}$ and $M_{0}^{p}$ are equivalent to certain Herz spaces (respectively, inhomogeneous and homogeneous), where pointwise convergence of approximate identities holds by the results of [9] and [10]. For the Marcinkiewicz spaces, instead, only a weak sufficient condition for pointwise convergence of approximate identities is known [2], based on a rather strong decay condition: counterexamples are available when this condition fails.

In Sect. 3 we show that on all these spaces the approximate identities behave quite differently than on $L^{p}$ in a very strong sense: there exist functions $f$ in all these spaces such that all approximate identities $\phi_{\varepsilon} * f$ converge to $f$ pointwise almost everywhere as $\varepsilon \rightarrow 0$, but $\phi_{\varepsilon} * f$ does not converge to $f$ in norm (or semi-norm). By the results of Sect. 2 we know that, in these spaces, for every approximate identity there is some function $f$ such that $\phi_{\varepsilon} * f$ does not converge to $f$ in norm (or semi-norm): but we construct an $f$ such that norm convergence at $f$ fails for all approximate identities, and nevertheless pointwise convergence almost everywhere holds.

Because of the recent results on pointwise convergence quoted above (some of which obtained at the same time as ours, but independently) pointwise convergence is known for all these bounded mean spaces except $\mathcal{M}^{p}$ and $\widetilde{\mathcal{M}}^{p}$. So the most interesting space in what follows is the Marcinkiewicz space, where pointwise convergence does not always hold, and its Banach quotient $\widetilde{\mathcal{M}}^{p}$. Here is their definition. 
Marcinkiewicz spaces $\mathcal{M}^{p}(\mathbb{R})$ were introduced in [4] and studied in [3,5,12-14] and [2]. $\mathcal{M}^{p}(\mathbb{R})$ is defined as the space of functions $f \in L^{p}$ on all compact sets in $\mathbb{R}, 1 \leqslant p<\infty$, such that

$$
\limsup _{T \rightarrow+\infty} \frac{1}{2 T} \int_{-T}^{T}|f(s)|^{p} d s<+\infty .
$$

We equip $\mathcal{M}^{p}(\mathbb{R}), 1 \leqslant p<+\infty$, with the following seminorm:

$$
\|f\|_{\mathcal{M}^{p}}^{p}=\limsup _{T \rightarrow+\infty} \frac{1}{2 T} \int_{-T}^{T}|f(s)|^{p} d s .
$$

$\mathcal{M}^{p}(\mathbb{R})$ is complete with respect to this seminorm for $1 \leqslant p<+\infty$ [14]. The quotient of $\mathcal{M}^{p}$ with respect to the null space $\mathcal{I}^{p}$ of the seminorm is therefore a Banach space, which we denote by $\widetilde{\mathcal{M}}^{p}$.

It is obvious that translations are isometries in $\mathcal{M}^{p}$.

It has been observed in [3] that all regular bounded Borel measures give rise to bounded convolution operators on $\mathcal{M}^{p}(\mathbb{R})$. In particular, if $\phi \in L^{1}(\mathbb{R})$, for every $f \in \mathcal{M}^{p}$ one has

$$
\|\phi * f\|_{\mathcal{M}^{p}} \leqslant\|\phi\|_{1}\|f\|_{\mathcal{M}^{p}} .
$$

Therefore $\mathcal{M}^{p}$ is a Banach convolution module over $L^{1}(\mathbb{R})$.

Another interesting family of locally summable function spaces is given by the Stepanoff spaces, also introduced in [4] and studied in [1,3,5]. Stepanoff functions are those measurable functions whose $L^{p}$-norm in intervals of length, say, 1 , is bounded. The supremum of these norms, $\sup _{x \in \mathbb{R}} \int_{x}^{x+1}|f|^{p}$, defines a norm for the Stepanoff space $\mathcal{S}^{p}, 1 \leqslant p<\infty$. The Stepanoff space $\mathcal{S}^{\infty}$ coincides with $L^{\infty}$ and is not considered in this paper. It is clear that $L^{p}(\mathbb{R})$ is contained in $\mathcal{S}^{p},\|f\|_{\mathcal{S}^{p}} \leqslant\|f\|_{p}$ and $\mathcal{S}^{p}$ is complete. Translations are isometries in $\mathcal{S}^{p}$, obviously. It can be seen, as in the case of $\mathcal{M}^{p}$, that $L^{1}$ functions, and more generally bounded Borel measures, are bounded convolution operators in the Stepanoff norm, and $\mathcal{S}^{p}$ is a Banach module over $L^{1}$ :

$$
\|\phi * f\|_{\mathcal{S}^{p}} \leqslant\|\phi\|_{1}\|f\|_{\mathcal{S}^{p}} .
$$

For the details, see $[6,11]$ (in the latter reference, the Stepanoff spaces are a particular case of weighted Wiener amalgams, namely $\left.W\left(L^{p}, \ell^{\infty}\right)\right)$.

A family of spaces with norms related to the Marcinkiewicz and Stepanoff norms are the bounded $p$-mean spaces $M^{p}$, introduced again in [4] and studied in [12] and [8]. Their norm is defined as

$$
\|f\|_{M^{p}}^{p}=\sup _{T>1} \frac{1}{2 T} \int_{-T}^{T}|f(s)|^{p} d s .
$$


Obviously, for every $K>0$ this norm is equivalent to the norm of the dilated space $M_{K}^{p}$ defined by

$$
\|f\|_{M_{K}^{p}}^{p}=\sup _{T>K} \frac{1}{2 T} \int_{-T}^{T}|f(s)|^{p} d s .
$$

Instead, as we shall see, this norm is inequivalent to the corresponding norm for $K=0$ : the space $M_{0}^{p}$ is not isomorphic to $M_{K}^{p}$.

Since $\|f\|_{\mathcal{M}^{p}} \leqslant\|f\|_{M^{p}}$, the space $M^{p}$ embeds continuously in $\mathcal{M}^{p}$, but the embedding is not an isomorphism.

\section{Translations and approximate identities}

As for the spaces introduced before, also $M^{p}$ is complete. However, translations are not isometries here (see also [6]):

Lemma 2.1 The translation operator $\lambda_{x}$ is bounded on $M_{K}^{p}$, with norm $w(x) \equiv$ $\left|\left\|\lambda_{x}\right\|\right| \geqslant 1+\frac{|x|}{K}$.

Proof Without loss of generality, let $x>0$. Choose $0<\eta<x$ and let $\chi$ be the characteristic function of the interval $[x-\eta, x+\eta]$. Suppose that $K \leqslant x+\eta$. Then, for $T \geqslant K$, the $p$-mean $\frac{1}{2 T} \int_{-T}^{T}|\chi(s)|^{p} d s$ is largest for $T=x+\eta$, and its maximum value $\|\chi\|_{M_{K}^{p}}$ is $(\eta /(x+\eta))^{1 / p}$. On the other hand, the translate $\lambda_{x} \chi$ is a characteristic function centered at 0 , and its norm in $M_{K}^{p}$ is 1 if $\eta \geqslant K$ and $(\eta / K)^{1 / p}$ otherwise. Therefore

$$
\left\|\lambda_{x} \chi\right\|_{M_{K}^{p}}=\frac{x+\eta}{\max \{\eta, K\}}\|\chi\|_{M_{K}^{p}} .
$$

The constant in this inequality is largest for $\eta=K$ and its largest value is $1+\frac{x}{K}$.

This lemma and the obvious inequality $\|f\|_{M^{p}} \leqslant\|f\|_{M_{0}^{p}}$ yield:

Corollary 2.2 If $x \neq 0$, the translation operator $\lambda_{x}$ is unbounded on $M_{0}^{p}$. In particular, the Banach space $M_{0}^{p}$ embeds continuously in $M^{p}$ but the embedding is not a Banach space isomorphism.

Because translations are not isometries in $M^{p}$, these spaces are not Banach modules over $L^{1}$. Instead:

Remark 2.3 Let $w(x)=\left|\left\|\lambda_{x}\right\|\right|$ as in Lemma 2.1, and denote by $L_{w}^{1}$ the weighted $L^{1}$ space with norm $\|\phi\|_{1, w}=\int_{-\infty}^{\infty}|\phi(t)| w(t) d t$. Then $M^{p}$ is a Banach module over $L_{w}^{1}$, that is, for every $\phi \in L_{w}^{1}$ and $f \in M^{p}$ the convolution $\phi * f$ is defined (because $\left.M^{p} \subset \mathcal{M}^{p}\right)$, and

$$
\|\phi * f\|_{M^{p}} \leqslant\|\phi\|_{1, w}\|f\|_{M^{p}}
$$


This fact is well known (see, for instance, [8, inequality (30)]). Since its proof is very short, we rewrite it here for the sake of completeness:

$$
\begin{aligned}
\|\phi * f\|_{M^{p}} \leqslant \int_{-\infty}^{\infty}|\phi(x)|\left\|\lambda_{x} f\right\|_{M^{p}} d x & \leqslant\|f\|_{M^{p}} \int_{-\infty}^{\infty}|\phi(x)| w(x) d x \\
& =\|\phi\|_{1, w}\|f\|_{M^{p}} .
\end{aligned}
$$

A similar weight function does not exist for $M_{0}^{p}$ : it is infinite. Therefore we expect that this space is not a module over a weighted $L^{1}$ space. More than that is true:

Proposition 2.4 Compactly supported $L^{1}$ functions convolve $M_{0}^{p}$ into $M^{p}$ : in particular, the convolution $\phi * f$ exists almost everywhere for all compactly supported $\phi \in L^{1}$ and for every $f \in M_{0}^{p}$. The same is true for all $\phi \in L_{w}^{1}$, the Beurling algebra associated with the weight functions $w$ defined by the norm of translations on $M^{p}$. However, there exist $f \in M_{0}^{p}$ such that

$$
\sup \left\{\frac{\|\phi * f\|_{M_{0}^{p}}}{\|f\|_{M_{0}^{p}}}:\|\phi\|_{1}=1\right\}=\infty
$$

Proof If $\phi \in L_{w}^{1}$ the convolution belongs to $M^{p}$ because $M_{0}^{p} \subset M^{p}$ and this space is a module over $L_{w}^{1}$ : in particular this is true for compactly supported $L^{1}$ functions, because they belong to $L_{w}^{1}$ (here of course $w$ is the weight function constructed above for $M^{p}$, not for $M_{0}^{p}$ ). The norm inequality is given by the same function that we used in Lemma 2.1 to prove unboundedness of translations in $M_{0}^{p}$. More precisely, fix $x \neq 0$ and (with a slight notation change) let now $\chi_{\eta}$ be the characteristic function of an interval of width $\eta$ centered at $x$; let $\phi(t)=\frac{1}{\eta} \chi_{\eta}(-t)$. Then $\|\phi\|_{1}=1$ and $\left\|\chi_{\eta}\right\|_{M_{0}^{p}}=\left(\frac{\eta}{2 x+\eta}\right)^{1 / p}$, but the graph of $\phi * \chi_{\eta}$ is a triangle of width $2 \eta$ and height 1 centered at 0 , and so the supremum of its $p$-means on $[-T, T]$ is attained for small $T$ and has value $\left\|\phi * \chi_{\eta}\right\|_{M_{0}^{p}}=1$. Therefore, when $\eta$ becomes small, the ratio $\left\|\phi * \chi_{\eta}\right\|_{M_{0}^{p}} /\left\|\chi_{\eta}\right\|_{M_{0}^{p}}$ diverges.

Therefore it makes sense to consider approximate identities defined by $L^{1}$ functions on $\mathcal{M}^{p}, \widetilde{\mathcal{M}}{ }^{p}, \mathcal{S}^{p}$ and by $L_{w}^{1}$ functions on $M^{p}$ and $M_{0}^{p}$.

The following result is a particular case of [7, Proposition 1.1].

Proposition 2.5 Let $B$ be a normed or semi-normed space of functions on $\mathbb{R}$ where translations are continuous operators, and let $w(x)=\left|\left\|\lambda_{x}\right\|\right|$. Let $B$ be a Banach module on the weighted $L^{1}$ space $L_{w}^{1}(\mathbb{R})$ and $f \in B$. Approximate identities in $L_{w}^{1}$ applied to $f$ converge in norm (that is, for every $\varepsilon>0$ there is $g \in L_{w}^{1}$ such that $\|g * f-f\|_{B}<\varepsilon$ ) if and only if translation is strongly continuous on $f: \lim _{x \rightarrow 0}\left\|\lambda_{x} f-f\right\|_{B}=0$.

Proposition 2.6 Translation is not strongly continuous in $\mathcal{M}^{p}, \mathcal{S}^{p}, M^{p}$ and $M_{0}^{p}$. 
Proof Let $f(x)=\sum_{n=0}^{\infty} 2^{n / p} \chi_{\left[n, n+2^{-n}\right]}(|x|)$ (here $\chi$ denotes the characteristic function). It is easy to verify that $\|f\|_{\mathcal{M}^{p}}=1$ and $\|f\|<2$ in the other three spaces. For $0<x<1$, the right translation $\lambda_{x} f$ has support disjoint from $f$ when restricted to the positive half-line $L_{+}=\left\{t \geqslant n\right.$ where $n$ is the smallest integer such that $\left.2^{-n} \leqslant x\right\}$, that is, $L_{+}=\left\{t: 2^{-t} \leqslant x\right\}$; the same happens to left translations on a negative half-line $L_{-}$. Denote by $f_{ \pm}$the restrictions of $f$ to $L_{ \pm}$, respectively. Now, if $x>0$, and $B$ denotes any of the spaces in the statement,

$$
\left\|\lambda_{x} f-f\right\|_{B}^{p}>\left\|\lambda_{x} f_{+}-f_{+}\right\|_{B}^{p} .
$$

If $f$ belongs to $\mathcal{M}^{p}$, since the supports of $f_{+}$and $\lambda_{x} f_{+}$are disjoint, the right hand side is

$$
\begin{aligned}
\left\|\lambda_{x} f_{+}-f_{+}\right\|_{\mathcal{M}^{p}}^{p} & =\underset{T}{\limsup } \frac{1}{2 T}\left(\int_{-T}^{T}\left|\lambda_{x} f_{+}-f_{+}\right|^{p}\right) \\
& =\underset{T}{\limsup } \frac{1}{2 T}\left(\int_{-T}^{T}\left|\lambda_{x} f_{+}\right|^{p}+\int_{-T}^{T}\left|f_{+}\right|^{p}\right) \\
& =\underset{T}{\limsup } \frac{1}{2 T} \int_{-T}^{T}\left|f_{+}\right|^{p}=2\left\|f_{+}\right\|_{\mathcal{M}^{p}}^{p} .
\end{aligned}
$$

If $B=M^{p}$ or $M_{0}^{p}$ and $f \in B$, the same argument yields

$$
\begin{aligned}
\left\|\lambda_{x} f_{+}-f_{+}\right\|_{B}^{p} & =\sup _{T} \frac{1}{2 T} \int_{-T}^{T}\left|\lambda_{x} f_{+}-f_{+}\right|^{p} \\
& =\sup _{T} \frac{1}{2 T}\left(\int_{-T}^{T}\left|\lambda_{x} f_{+}\right|^{p}+\int_{-T}^{T}\left|f_{+}\right|^{p}\right)>\left\|f_{+}\right\|_{B}^{p} .
\end{aligned}
$$

Finally, if $f \in \mathcal{S}^{p}$, then for $x>0$ one has

$$
\left\|\lambda_{x} f_{+}-f_{+}\right\|_{\mathcal{S}^{p}}^{p}=\sup _{y} \int_{y}^{y+1}\left(\left|\lambda_{x} f_{+}\right|^{p}+\left|f_{+}\right|^{p}\right)>\sup _{y} \int_{y}^{y+1}\left|f_{+}\right|^{p}=\left\|f_{+}\right\|_{\mathcal{S}^{p}}^{p},
$$

again by disjointness of the supports. The same inequalities hold for $x<0$ by restricting to $L_{-}$. In all cases, $\lambda_{x} f \nrightarrow f$ as $x \rightarrow 0$.

By Propositions 2.5 and 2.6 we know that approximate identities do not converge in the norm of $\mathcal{M}^{p}, \widetilde{\mathcal{M}}^{p}, \mathcal{S}^{p}$ and $M^{p}$. Remark 2.4 suggests that the same should be 
true for $M_{0}^{p}$. In Sect. 3 we prove a more surprising fact: in each of these spaces there is a function $f$ such that no approximate identities $\phi_{\varepsilon} * f$ converge to $f$ in the seminorm of $\mathcal{M}^{p}$ or in the norm of the other spaces (Theorem 3.1 and Proposition 3.5), but all converge pointwise. For this we need a bounded function $f$ : therefore the function used in Proposition 2.6 to show that translation is not strongly continuous is not enough.

General sufficient conditions for pointwise convergence of approximate identities have been obtained in [9] for a class of spaces equivalent to Stepanoff spaces (namely, Wiener amalgams); moreover, the spaces $M^{p}$ and $M_{0}^{p}$ are equivalent to certain Herz spaces (respectively, inhomogeneous and homogeneous), where pointwise convergence of approximate identities holds by the results of [9] and [10].

\section{Strong convergence of approximate identities}

Theorem 3.1 There exists a function $f \in \mathcal{M}^{p}$ such that, for all normalized non-negative $\phi \in L^{1}, \lim _{\varepsilon \rightarrow 0^{+}} \phi_{\varepsilon} * f-f$ is not zero in the seminorm of $\mathcal{M}^{p}$, but is zero almost everywhere. For $\varepsilon \rightarrow 0^{+}, \phi_{\varepsilon} * f$ does not converge in measure.

Proof We first show that the result holds when $\phi$ is the characteristic function of $[0,1]$. Then we extend the result to all step functions, and finally, by approximation, to all $L^{1}$ functions. So, let us now set $\phi=\chi_{[0,1]}$. We proceed to construct a function $f \in \mathcal{M}^{p}$ such that $\left\|\phi_{\varepsilon} * f-f\right\|_{\mathcal{M}^{p}} \nrightarrow 0$ as $\varepsilon \rightarrow 0^{+}$. We take $f(x)=0$ if $x<0, f(x)=1$ if $0 \leqslant x<\frac{1}{2}, f(x)=-1$ if $\frac{1}{2} \leqslant x<1$, and then we double the oscillation frequency in each subsequent interval $[n, n+1)$, that is, define $f$ as follows. Let $\zeta(x)=x-[x]$ and $f_{0}(x)=f(\zeta(x))$, and for $n \leqslant x<n+1$ let $f(x)=f_{0}\left(2^{n} x\right)$. Observe that in the interval $[n, n+1)$ the functions $f$ swaps $2^{n+1}$ times between the values \pm 1 , and so it is constant on adjacent subintervals of length $\delta=2^{-n-1}$. Since $|f| \equiv 1$, it is clear that $\|f\|_{\mathcal{M}^{p}}=1$.

Let us now choose $\varepsilon=2^{-k}$ and $n>k$, and let us compute the convolution of $\phi_{\varepsilon}$ with the restriction of $f$ to $[n, n+1)$. To this goal, we first compute the convolution of $\phi_{\varepsilon}$ with the characteristic function $\chi$ of $[n, n+\delta)$, where $\delta$ is as above. The following facts are geometrically obvious, and elementary to verify: $\phi_{\varepsilon} * \chi$ vanishes if $x \leqslant n-\varepsilon$ or $x \geqslant n+\delta$ (by disjointness of supports); moreover, $\phi_{\varepsilon} * \chi$ has value $\frac{\delta}{\varepsilon}$ in $n-\varepsilon+\delta \leqslant x \leqslant n$ (because $\phi_{\varepsilon}$ has constant value $1 / \varepsilon$ on its support, and for these values of $x$, the support of $\chi$, of length $\delta$, is contained in the support of the translate $\lambda_{x} \phi_{\varepsilon}$ ); finally, $\phi_{\varepsilon} * \chi(x)$ is the linear interpolation between the extreme values 0 and $\frac{\delta}{\varepsilon}$ in the intervals $[n-\varepsilon, n-\varepsilon+\delta]$ and $[n, n+\delta]$.

Now it is easy to compute the convolution of $\phi_{\varepsilon}$ with the one-cycle zero-average square wave $\theta$ whose nonzero values are 1 on $[n, n+\delta)$ and -1 on $[n+\delta, n+2 \delta)$ : that is, $\theta(x)=f_{0}(x / 2 \delta)$ for $n \leqslant x<n+\delta$ and 0 otherwise. Indeed, $\phi_{\varepsilon} * \theta$ is the function whose graph consists only of the following two triangles of basewidth $2 \delta$ and height $\frac{\delta}{\varepsilon}$ : the first positive (that is, above the $\mathrm{x}$-axis) in the interval $[n-\varepsilon, n-\varepsilon+2 \delta]$ (i.e., the equilateral triangle with base on the $x$-axis and apex at $x=n-\varepsilon+\delta$, $y=\frac{\delta}{\varepsilon}$ ), and the second negative in the interval $[n, n+2 \delta]$ (with apex at $x=n+\delta$, $\left.y=-\frac{\delta}{\varepsilon}\right)$. More explicitly, let $t$ be the function with triangular graph defined on $[-1,1]$ 
by $t(x)=1+x$ if $-1 \leqslant x \leqslant 0$ and $t(x)=1-x$ if $0 \leqslant x \leqslant 1$, and let $\tau(x)=t(x / \delta)$. Then $\phi_{\varepsilon} * \theta=\frac{\delta}{\varepsilon} \kappa$, where

$$
\kappa(x)= \begin{cases}\tau(x-(n-\varepsilon+\delta)) & \text { if } x \in[n-\varepsilon, n-\varepsilon+2 \delta] \\ \tau(x-(n+\delta)) & \text { if } x \in[n, n+2 \delta] \\ 0 & \text { otherwise }\end{cases}
$$

Finally, we make use of this observation to compute $\phi_{\varepsilon} * f_{n}$, where $f_{n}=f \chi_{[n, n+1]}$ is the sum of translates of step $2 \delta$ of the square wave $\theta$. Since $\varepsilon$ is a multiple of $2 \delta$, the triangular spikes of each of these translates cancel out except on the initial segment $[n-\varepsilon, n]$ and the final segment $[n+1, n+1+\varepsilon]$. In particular, $\phi_{\varepsilon} * f_{n}=0$ in $[n, n+1]$. It is important to observe that this fact does not depend on the choice of $\phi$, but only on the fact that $\phi$ is supported in an interval of length $\varepsilon$ and $f_{n}$ is the sum of consecutive translates of step $\delta$ of a function of average zero: since $\varepsilon$ is a multiple of $\delta$, then $f_{n}$ is a function of average zero on each interval of length $\varepsilon$ contained in $[n, n+1)$, that is on the corresponding translates of the support of $\phi_{\varepsilon}$. Now we return to our function $f=\sum_{n} f_{n}$. The supports of the sequence of functions $\phi_{\varepsilon} * f_{n}$ are not disjoint, but each of them overlaps only the support of the previous and of the next function of the sequence (because $\varepsilon<1$ ). So, by the previous observation, as $\varepsilon=2^{-k}$, in the half-line

$$
J=\left\{x \geqslant k=-\log _{2}(\varepsilon)\right\}
$$

one has $\phi_{\varepsilon} * f=0$ everywhere except in the intervals of radius $\varepsilon$ centered at the non-negative integers. Denote by $g$ the function supported on $J$ that vanishes in these intervals and coincides with $f$ outside. Since $|f| \equiv 1$, it is obvious that the $L^{p}$ averages are $\|g\|_{\mathcal{M}^{p}}=(1-2 \varepsilon)^{\frac{1}{p}}$. Thus

$$
\left\|\phi_{\varepsilon} * f-f\right\|_{\mathcal{M}^{p}} \geqslant\|g\|_{\mathcal{M}^{p}}=(1-2 \varepsilon)^{\frac{1}{p}} \rightarrow 1
$$

as $\varepsilon \rightarrow 0^{+}$. Therefore $\phi_{\varepsilon} * f$ does not converge to $f$ in seminorm.

We now extend these results to approximate identities generated by step functions. Observe that the argument outlined above also holds for every translate $\lambda_{y} \phi(x)=$ $\phi(x-y)$ : in this case, $\phi_{\varepsilon} * f=0$ in a translate of $[n+\varepsilon, n+1-\varepsilon]$ for every sufficiently large $n$, namely in $[n+\varepsilon+\varepsilon y, n+1-\varepsilon+\varepsilon y]$. But then, let $E=[-\varepsilon(1-y), \varepsilon(1+y)]$ and note that the measure of $E$ is $\rho=2 \varepsilon$. If, as before, $g$ is supported in $J$, vanishes in the intervals $n+E$ with $n \in \mathbb{N}$ and coincides with $f$ outside of these intervals, then every convex combination $\psi=\alpha \phi+(1-\alpha) \lambda_{y} \phi$ also satisfies

$$
\left\|\psi_{\varepsilon} * f-f\right\|_{\mathcal{M}^{p}} \geqslant\|g\|_{\mathcal{M}^{p}}=(1-2 \rho)^{\frac{1}{p}}
$$

Again, as $\rho=2 \varepsilon$, the right hand side tends to 1 as $\varepsilon \rightarrow 0^{+}$, hence the norm at the left hand side does not converge to zero. (The reader should observe that this inequality holds because $\psi_{\varepsilon} * f=f$ in appropriate intervals, and has nothing to do with the fact that translations be isometries.) 
Now we can extend this inequality to all step functions, that is all functions $\psi$ supported in a finite interval, say $[-T, T]$, that are finite convex combinations of characteristic functions of intervals. For every fixed $\rho>0$, choose $\varepsilon=\rho / T$ : then $\psi_{\varepsilon}$ has support in $[-\rho, \rho]$ and in the half-line $J$, by the same argument as above, $\psi_{\varepsilon}$ vanishes in the intervals $[n+\rho, n+1-\rho]$ : so (3) holds again.

Finally, let us deal with all $L^{1}$ approximate identities. Every $L^{1}$ function is an $L^{1}$ perturbation of step functions: for every $\xi \in L^{1}(\mathbb{R})$ and every $\eta>0$, there exist $T=T(\xi, \eta)$ and a step function $\psi$ with support in $[-T, T]$ such that $\|\xi-\psi\|_{1}<\eta$. Observe that $T \rightarrow+\infty$ if $\eta \rightarrow 0^{+}$. Let us write $\varepsilon=\frac{1}{4 T}$ : note that $\varepsilon \rightarrow 0^{+}$ when $\eta \rightarrow 0^{+}$. Now $\psi_{\varepsilon}$ is supported in $\left[-\frac{1}{4}, \frac{1}{4}\right]$, and, by (3), $\psi_{\varepsilon} * f=0$ in all the intervals $I_{n}=\left[n+\frac{1}{4}, n+\frac{3}{4}\right] \subset J$. Observe that, by (1), the half-line $J$ begins at the point $n_{0}=-\log _{2}(\varepsilon)$ that depends only on $\xi$ and $T$, hence on $\eta$. Since the increasing frequency square wave $f$ has absolute value 1 , every $h \in L^{1}(\mathbb{R})$ satisfies $\|h * f\|_{\infty} \leqslant\|h\|_{1}$. But then, for $x>n_{0}$,

$$
\xi_{\varepsilon} * f=(\xi-\psi)_{\varepsilon} * f+\psi_{\varepsilon} * f<\eta
$$

in all the intervals $I_{n}, n>n_{0}$. As $|f|=1$, now $\left|\xi_{\varepsilon} * f-f\right|>1-\eta$ in these intervals, and $\left\|\xi_{\varepsilon} * f-f\right\|_{\mathcal{M}^{p}} \geqslant 2^{-\frac{1}{p}}(1-\eta)$. Therefore the argument holds for all non-negative functions $\xi$ with integral 1 .

We now consider pointwise convergence, that is easily proved as follows. Let $\xi \in L^{1}$. If we fix $n$ and let $\varepsilon$ tend to zero (that is, $k \rightarrow+\infty$ ), then $\xi_{\varepsilon} * f$ converges pointwise to $f$. Indeed, this is true for $\xi_{\varepsilon} * f_{j}$ for every $j$, where $f_{j}=f \chi_{[j, j+1]}$, because $f_{j} \in L^{\infty}$. Now, $f=\sum_{j} f_{j}$, and if $n \leqslant x \leqslant n+1$, only $\phi_{\varepsilon} * f_{n}$ and $\phi_{\varepsilon} * f_{n \pm 1}$ do not vanish at $x$. Therefore $\phi_{\varepsilon} * f$ converges pointwise to $f$.

We have shown in (4) that, for every approximate identity $\xi_{\varepsilon}$ and for every $0<$ $\eta<1$, there is $\varepsilon_{\eta}>0$ such that, for $0<\varepsilon<\varepsilon_{\eta}$, one has $\left|\xi_{\varepsilon} * f\right|<\eta$ in $[n+\varepsilon$, $n+1-\varepsilon]$ if $2^{-n}<\varepsilon$. Since $|f| \equiv 1$, for every $\eta$ the measure of the set $\left\{x:\left(\xi_{\varepsilon} * f-f\right)\right.$ $(x)>1-\eta\}$ is infinite, and $\xi_{\varepsilon} * f$ does not converge to $f$ in measure (and no subsequence does, so $\xi_{\varepsilon} * f$ does not converge in measure to any limit).

Remark 3.2 For every $t \in \mathbb{R}$, the translation operator $\lambda_{t}$ is clearly isometric on $\mathcal{M}^{p}$. Therefore the map $t \mapsto \lambda_{t}$, from $\mathbb{R}$ to the space of bounded operators on $\mathcal{M}^{p}$, cannot be strongly continuous, otherwise approximate identities would converge in seminorm. The increasing frequency square wave function $f$ of the proof of Theorem 3.1 provides an example where $\lambda_{t} f$ does not converge to $f$ as $t \rightarrow 0$. Indeed, choose $t=2^{-k}$. It is easy to verify that $\lambda_{x} f=-f$ in the intervals $[n+t, n+1-t]$ for $n>k$. Therefore $\left\|\lambda_{t} f-f\right\|_{\mathcal{M}^{p}}=2$ for all such $t$.

Pointwise convergence almost everywhere of approximate identities in $\mathcal{M}^{p}$ has been proved in [2] when $\phi$ belongs to an appropriate subspace of $L^{1}$. Indeed, for $\phi \in L^{1}(\mathbb{R})$, consider the following least monotone majorant function:

$$
\psi_{\phi}(x)=\sup _{|t|>|x|}|\phi(t)|
$$


Usually $\psi_{\phi}$ does not belong to $L^{1}$ even though $\phi$ does: see [2] for an interesting example of a $\phi \in L^{1}$ with compact support such that $\psi_{\phi} \notin L^{1}$. On the other hand, it has been proved in [2] that, if $\phi \geqslant 0, \int \phi=1$ and $\psi_{\phi} \in L^{1}$ then $\phi_{\varepsilon} * f(x) \rightarrow f(x)$ at each point of continuity of $f$ as $\varepsilon \rightarrow 0^{+}$. Pointwise convergence almost everywhere of approximate identities on $\mathcal{M}^{p}$ in general fails without the condition $\psi_{\phi} \in L^{1}$ : an example given in [2]. Instead, our result holds for all normalized non-negative approximate identities $\phi \in L^{1}$.

The Marcinkiewicz space $\mathcal{M}^{p}$ is a seminormed space rather than a Banach space. This gives rise to a further ground of investigation: convergence of approximate identities is affected if one changes a Marcinkiewicz function by a null function modulo the null space $\mathcal{I}^{p}$ of the seminorm? Clearly, norm convergence cannot be affected. What about pointwise convergence? This is an important issue, necessary to transport pointwise convergence of approximate identities from $\mathcal{M}^{p}$ to its Banach quotient $\widetilde{\mathcal{M}}^{p}=\mathcal{M}^{p} / \mathcal{I}^{p}$.

In general, pointwise convergence of an approximate identity (that is, the fact that at a continuity point $x_{0}$ of $f$ the limit as $\varepsilon \rightarrow 0$ of $\phi_{\varepsilon} * f\left(x_{0}\right)-f\left(x_{0}\right)$ is 0$)$ is not invariant modulo the null space $\mathcal{I}^{p}$, as the next lemma shows. However, if $\phi(x)$ decays monotonically as $|x|$ grows, then its least monotone majorant $\psi$ belongs to $L^{1}(\mathbb{R})$, and so the approximate identity converges at every continuity point by the above mentioned result of [2]. This fact shows that the statement of Theorem 3.1 does not make sense for the quotient space $\widetilde{\mathcal{M}}^{p}$.

We follow an idea of [2]. Without loss of generality, we may assume that $x_{0}=0$ is a Lebesgue point: from now on we shall restrict attention to convergence at the point 0 . Let $\chi_{n}$ be the characteristic function of the interval $\left[2^{n}, 2^{n}+1\right]$, and let $\alpha_{n}>0$ be such that

$$
\sum_{n=0}^{+\infty} \alpha_{n}=1
$$

We set

$$
\phi=\sum_{n=0}^{+\infty} \alpha_{n} \chi_{n}
$$

Consider now the following function $f \in \mathcal{M}^{1}$ :

$$
f(x)=\sum_{n=0}^{+\infty} c_{n} \chi_{n}(|x|),
$$

where $c_{n}>0, c_{n}=O\left(2^{n}\right)$. Observe that

$$
\frac{1}{2^{n+1}} \int_{-2^{n}}^{2^{n}} f(x) d x<C \frac{1}{2^{n+1}} \sum_{k=0}^{n} 2^{k}<C,
$$

hence $f \in \mathcal{M}^{1}$. Similarly, if we define $f_{p}$ by choosing $c_{n}=O\left(2^{n / p}\right)$, then $f_{p} \in \mathcal{M}^{p}$. 
The following statement is [2, Proposition 3.1]:

Proposition 3.3 If $\sum_{m=0}^{+\infty} \alpha_{m} c_{m-n}=+\infty$, then it is not true that $\phi_{\varepsilon} * f(0) \rightarrow f(0)$ as $\varepsilon \rightarrow 0^{+}$. The condition is satisfied, for instance, if $\alpha_{m}=2^{-m}$ and $c_{m}=2^{m}$.

Corollary 3.4 Let $\mathcal{I}^{p}$ be the null space of the seminorm of $\mathcal{M}^{p}$. Let $\phi \in L^{1}(\mathbb{R})$, with $\int_{-\infty}^{\infty} \phi=1$. If $f \in \mathcal{M}^{p}$ satisfies $\left\|\lambda_{x}(f)-f\right\|_{\mathcal{M}^{p}} \rightarrow 0$ as $x \rightarrow 0$, then $\left\|\phi_{\varepsilon} * g-g\right\|_{\mathcal{M}^{p}} \rightarrow 0$ as $\varepsilon \rightarrow 0^{+}$for every $g \in \mathcal{M}^{p}$ such that $g-f \in \mathcal{I}^{p}$. On the other hand, if $\phi_{\varepsilon} * f(x) \rightarrow f(x)$ almost everywhere as $\varepsilon \rightarrow 0^{+}$, then in general it is not true that, for every other $g \in \mathcal{M}^{p}$ such that $g-f \in \mathcal{I}^{p}$, one has $\phi_{\varepsilon} * g(x) \rightarrow g(x)$ almost everywhere.

Proof The first half of the statement follows obviously by expressing the convolution as an integral of translations. For the second half, it is enough to produce a function $f \in \mathcal{I}^{p}$ such that $\phi_{\varepsilon} * f(x)-f(x)$ does not tend to 0 as $\varepsilon \rightarrow 0^{+}$. For this, take the function $f(x)=\sum_{n=0}^{+\infty} c_{n} \chi_{n}(|x|)$ of the proof of Proposition 3.3, with $c_{n}=2^{n} / n$. If $\alpha_{n}=2^{-n}$, then $\sum_{m=0}^{+\infty} \alpha_{m} c_{m-n}=+\infty$, and so, by the Proposition, pointwise convergence does not hold almost everywhere. On the other hand, choosing for simplicity $n=2 m$ even, we have

$$
2^{-n-1} \sum_{k=0}^{n} \frac{2^{k}}{k} \leqslant 2^{-n-1}\left(\sum_{k=0}^{m} 2^{k}+\frac{1}{m} \sum_{k=m+1}^{2 m} 2^{k}\right)=2^{m-n}+\frac{1}{m}=2^{-\frac{n}{2}}+\frac{2}{n},
$$

and so

$$
\|f\|_{\mathcal{M}^{p}}=\lim _{n \rightarrow \infty} 2^{-n-1} \sum_{k=0}^{n} \frac{2^{k}}{k}=0 .
$$

The fact that approximate identities are pointwise convergent but not norm convergent is not a peculiarity of $\mathcal{M}^{p}$ only: the next statement extends this fact to the other spaces of bounded $L^{p}$ averages considered in this paper.

Proposition 3.5 The statement of Theorem 3.1 holds also for the spaces $\mathcal{S}^{p}, M^{p}$ and $M_{0}^{p}$.

Proof Let $B$ be one of the spaces in the statement. We extend the proof already given for $B=\mathcal{M}^{p}$. The argument for pointwise convergence of course has nothing to do with the choice of norm, hence of $B$. The lack of convergence in norm holds for all these spaces $B$ for the same reason as for $\mathcal{M}^{p}$. Indeed, the function $f$ introduced in the proof of Theorem 3.1 has modulus 1, hence it belongs to all these spaces. We have shown in that proof that, for $n$ appropriately large (depending on $\varepsilon$ ), one has $\phi_{\varepsilon} * f=0$ in $J_{n, \varepsilon}=[n+\varepsilon, n+1-\varepsilon]$ (here $\phi=\chi_{[0,1]}$, as at the beginning of the proof of Theorem 3.1). Since $J_{n, \varepsilon}$ exhausts $[n, n+1]$ as $\varepsilon$ tends to $0^{+}$, this implies that $\phi_{\varepsilon} * f \nrightarrow f$ in any of the norms. The extension to all approximate identities and the lack of convergence in measure are proved as in Theorem 3.1. 


\section{References}

1. Andreano, F., Grande, R.: Maximal characterization of locally summable functions. J. Anal. Appl. 26, 1-7 (2007)

2. Andreano, F., Grande, R.: Convolution on spaces of locally summable functions. J. Funct. Spaces Appl. 6, 187-203 (2008)

3. Bertrandias, J.: Espaces de fonctions bornées et continues en moyenne asympotique d'ordre $p$. Bull. Soc. Math. France 5, 1-106 (1966)

4. Besicovitch, A.S.: Almost Periodic Functions. Cambridge University Press, Cambridge (1932)

5. Bohr, H., Fölner, E.: On some type of functional spaces. Acta Math. 76, 31-155 (1944)

6. Feichtinger, H.G.: Banach convolution algebras of Wiener type. Symp. Math. XXIX, 267-301 (1985)

7. Feichtinger, H.G.: Compactness in translation invariant Banach spaces of distributions and compact multipliers. J. Math. Anal. Appl. 102, 289-327 (1984)

8. Feichtinger, H.G.: An elementary approach to Wiener's third Tauberian theorem for the Euclidean $n$-space. In: Proc. Conf. on Functions, Series, Operators, Budapest 1980. Coll. Math. Soc. Janos Bolyai, vol. 35, pp. 509-524. North-Holland, Amsterdam (1983)

9. Feichtinger, H.G., Weisz, F.: Wiener amalgams and pointwise summability of Fourier transforms and Fourier series. Math. Proc. Camb. Philos. Soc. 140, 509-536 (2006)

10. Feichtinger, H.G., Weisz, F.: Herz spaces and summability of Fourier transforms. Math. Nachr. Camb. Philos. Soc. 281, 309-324 (2008)

11. Heil, C.: An introduction to weighted Wiener amalgams. In: Krishna, M., Radha, R., Tanghavelu, S. (eds.) Wavelets and Their Applications, pp. 183-216. Allied Publishers, New Delhi (2003)

12. Lau, K.S.: On the Banach spaces of functions with bounded upper means. Pacif. J. Math. 91, 153-173 (1980)

13. Lau, K.S.: The class of convolution operators on the Marcinkiewicz spaces. Ann. Inst. Fourier 31, 225-243 (1981)

14. Marchinkiewicz, J.: Un remarque sur les éspace de A.S. Besicovitch. C. R. Acad. Sci. Paris 208, 157-159 (1939)

15. Shapiro, H.S.: Topics in Approximation Theory. Lecture Notes in Math., vol. 187. Springer, Berlin (1971) 